\title{
New Distance Measures between the Interval-Valued Complex Fuzzy Sets with Applications to Decision-Making
}

\author{
Haifeng Song, ${ }^{1}$ Lvqing $\mathrm{Bi}^{2}{ }^{\mathrm{Bo}} \mathrm{Hu}^{3}{ }^{3}$ Yingying $\mathrm{Xu}^{1},{ }^{1}$ and Songsong Dai ${ }^{1}$ \\ ${ }^{1}$ School of Electronics and Information Engineering, Taizhou University, Taizhou 318000, China \\ ${ }^{2}$ School of Electronics and Communication Engineering, \\ Guangxi Colleges and Universities Key Laboratory of Complex System Optimization and Big Data Processing, Yulin Normal \\ University, Yulin 537000, China \\ ${ }^{3}$ School of Mechanical and Electrical Engineering, Guizhou Normal University, Guiyang 550025, China
}

Correspondence should be addressed to Songsong Dai; ssdai@tzc.edu.cn

Received 16 October 2020; Revised 15 December 2020; Accepted 23 February 2021; Published 5 March 2021

Academic Editor: Harish Garg

Copyright ( 2021 Haifeng Song et al. This is an open access article distributed under the Creative Commons Attribution License, which permits unrestricted use, distribution, and reproduction in any medium, provided the original work is properly cited.

As a generalization of complex fuzzy set (CFS), interval-valued complex fuzzy set (IVCFS) is a new research topic in the field of CFS theory, which can handle two different information features with the uncertainty. Distance is an important tool in the field of IVCFS theory. To enhance the applicability of IVCFS, this paper presents some new interval-valued complex fuzzy distances based on traditional Hamming and Euclidean distances of complex numbers. Furthermore, we elucidate the geometric properties of these distances. Finally, these distances are used to deal with decision-making problem in the IVCFS environment.

\section{Introduction}

Since Ramot et al. [1] introduced complex fuzzy set (CFS) as a generalization of the classical fuzzy sets (FSs) in 2002, and CFS and its generations including interval-valued complex fuzzy set (IVCFS), complex intuitionistic fuzzy set, complex Pythagorean fuzzy set, complex picture fuzzy set, and complex q-rung orthopair fuzzy set have been successfully applied to many domains such as time series prediction [2-5], decision-making [6-10], signal processing [11-14], and image restoration [15]. Distance is an important tool in both theory and application of CFSs. Several distances between CFSs have been proposed [12, 16-19]. However, when CFSs are used to address uncertainty of target's position, distances in $[12,16,17]$ are not suitable; for instance, $\varepsilon$. $e^{j 0.25 \pi}$ and $\varepsilon \cdot e^{j 1.25 \pi}$ are near for any small number $\varepsilon>0$ where $j=\sqrt{-1}$, as shown in Figure 1 , but by using the method in [12], their distance is $\max (|0.01-0.01|,(|0.25 \pi-1.25 \pi| / 2 \pi))=0.5 \quad$ when $\varepsilon=0.01$. This is inconsistent with our vision. The main reason is that distances in $[12,16,17]$ are combining the difference between the amplitude terms and the difference between the phase terms of CFSs. This method ignored the circular structure of CFS. Two CFSs around the center can arbitrarily approach to each other, but their phase terms are completely opposite with the biggest difference. This causes the result, which is not consistent with our intuition. In this environment, using traditional distance between complex number is a more reasonable selection for us to measure the difference between CFSs.

Greenfield et al. [20, 21] introduced the IVCFS theory. In real life, when we get some answers such as " $0.5 \mathrm{~km}-0.6 \mathrm{~km}$, east" and " $0.5 \mathrm{~km}-0.7 \mathrm{~km}$, northwest" about the targets, we can represent these answers in terms of IVCFSs. Then, we may ask the simple question: what is the distance between " $0.5 \mathrm{~km}-0.6 \mathrm{~km}$, east" and "0.5 km-0.7 km, northwest" (see Figure 2)? Dai et al. [22] proposed some distance measures between IVCFSs. When IVCFSs are reduced to CFSs, this inevitably leads to get the same result in the above instance of Figure 1. Therefore, distances in [22] cannot overcome the above drawback of distances of CFSs and are not suitable for IVCFSs in some cases.

The main contribution to this article is summarized as follows: 


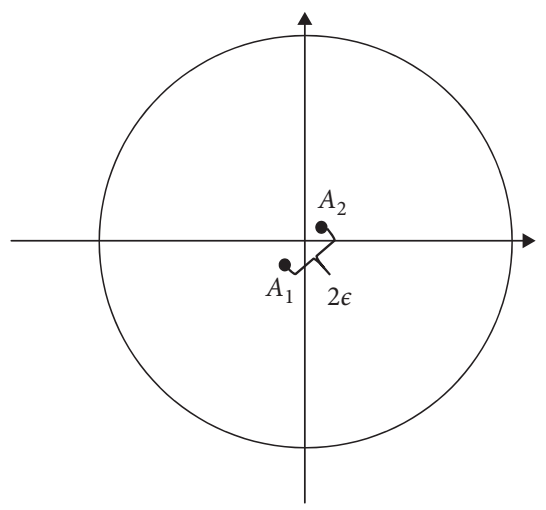

Figure 1: Distance of $A_{1}$ and $A_{2}$.

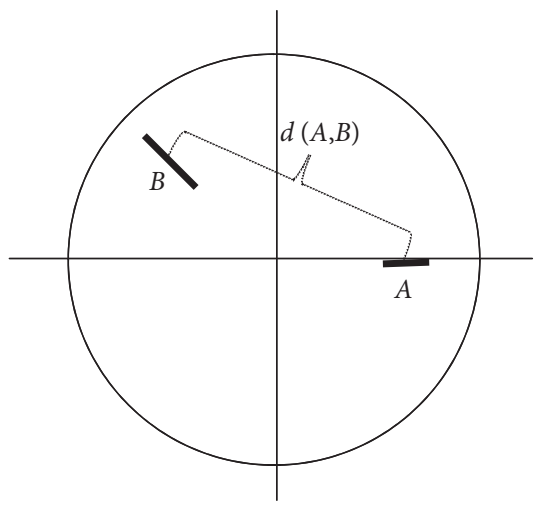

FIgURE 2: Distance.

(1) Some new distances for IVCFSs are constructed. They can overcome the above drawback of distances in IVCFSs. These distances also are new measures for CFS.

(2) Rotational invariance and reflectional invariance of these distances are studied, and a comparative analysis is provided.

(3) These distances are used in target selection problem when IVCFSs express the locative information of targets.

The purpose of this paper is to construct some distances between IVCFSs and apply them into decision-making problem. This article is structured as follows. In Section 2, we introduce the concept of IVCFS. In Section 3, we present some distances for IVCFSs. In Section 4, the rotational invariance and reflectional invariance of our proposed distances are studied. In Section 5, these distances are applied to solve a decision-making problem in IVCFSs information. In Section 6, a conclusion is given.

\section{Preliminaries}

In this paper, our discussion is based on IVCFSs. We first recall some basic concepts [1, 20, 21, 23-27].

Let $\bar{D}=\{c \in \mathbb{C}|| c \mid=1\}$ and $\rrbracket^{[0,1]}=\{[a, b] \mid 0 \leq a \leq b \leq 1\}$. Let $S$ be a fixed universe, then the following holds:

(1) A mapping $A: S \longrightarrow[0,1]$ is called a FS on $S$.

(2) A mapping $A: S \longrightarrow \square^{[0,1]}$ is called an interval-valued fuzzy set (IVFS) on $S$.

(3) A mapping $A: S \longrightarrow[0,1] \cdot \bar{D}$ is called a CFS on $S$.

(4) A mapping $A: S \longrightarrow \square^{[0,1]} \cdot \bar{D}$ is called an IVCFS on $S$.

Here, $\square^{[0,1]} \cdot \bar{D}$ is the dot product set of $\square^{[0,1]}$ and $\bar{D}$ and $\operatorname{IVCF}(S)$ is denoted as the set of all IVCFSs of $S$.

For any $s \in S$, its membership degree $\mu_{A}(s)$ is

$$
\left[\underline{p_{A}(s)}, \overline{p_{A}(s)}\right] \cdot e^{j q_{A}(s)} .
$$

For convenience, a value $a \in \mathbb{\natural}^{[0,1]} \cdot \bar{D}$ is called an interval-valued complex fuzzy value (IVCFV), denoted by $a=\left[p_{a}, \overline{p_{a}}\right] \cdot e^{j q_{a}}$.

For clarity, we list the membership functions for FS and its generalizations:

(1) For an IVFS $A$, its membership degree $\chi_{A}(s)$ is $\left[p_{A}(s), \overline{p_{A}(s)}\right]$.

(2) For a CFS $A$, its membership degree $\psi_{A}(s)$ is $p_{A}(s) \cdot e^{j q_{A}(s)}$.

(3) For a FS $A$, its membership degree $\eta_{A}(s)$ is $p_{A}(s)$.

\section{Distances between IVCFSs}

Definition 1 (see [22]). A function $d$ : $(\operatorname{IVCF}(S) \times$ $\operatorname{IVCF}(S)) \longrightarrow \mathbb{R}^{+} \cup\{0\}$ is called a distance measure between IVCFSs if it satisfies the following: for any $P, Q, R \in \operatorname{IVCF}(S)$,

(1) $d(P, Q) \geq 0$ and $d(P, Q)=0$ if and only if $P=Q$

(2) $d(P, Q)=d(Q, P)$

(3) $d(P, Q)+d(Q, R) \geq d(P, R)$

Dai et al. [22] defined the following distances in IVCFSs case as follows: for any $P, Q \in \operatorname{IVCF}(S)$, where $S=\left\{s_{1}, s_{2}, \ldots, s_{n}\right\}$,

$$
\begin{aligned}
& D_{H}(A, B)=\frac{1}{2} \sum_{i=1}^{n}\left(\frac{1}{2}\left|\underline{p_{A}\left(s_{i}\right)}-\underline{p_{B}\left(s_{i}\right) \mid}+\frac{1}{2}\right| \overline{p_{A}\left(s_{i}\right)}-\overline{p_{B}\left(s_{i}\right)}\left|+\frac{1}{2 \pi}\right| q_{A}\left(s_{i}\right)-q_{B}\left(s_{i}\right) \mid\right), \\
& D_{E}(A, B)=\sqrt{\mid \frac{1}{2} \sum_{i=1}^{n}\left(\frac{1}{2}\left|\underline{p_{A}\left(s_{i}\right)}-\underline{p_{B}\left(s_{i}\right)}\right|^{2}+\frac{1}{2}\left|\overline{p_{A}\left(s_{i}\right)}-\overline{p_{B}\left(s_{i}\right)}\right|^{2}+\frac{1}{4 \pi^{2}}\left|q_{A}\left(s_{i}\right)-q_{B}\left(s_{i}\right)\right|^{2}\right),}
\end{aligned}
$$




$$
\begin{aligned}
& D_{n H}(A, B)=\frac{1}{2 n} \sum_{i=1}^{n}\left(\frac{1}{2}\left|\underline{p_{A}\left(s_{i}\right)}-\underline{p_{B}\left(s_{i}\right) \mid}\right|+\frac{1}{2}\left|\overline{p_{A}\left(s_{i}\right)}-\overline{p_{B}\left(s_{i}\right)}\right|+\frac{1}{2 \pi}\left|q_{A}\left(s_{i}\right)-q_{B}\left(s_{i}\right)\right|\right), \\
& D_{n E}(A, B)=\sqrt{\frac{1}{2 n} \sum_{i=1}^{n}\left(\frac{1}{2}\left|\underline{p_{A}\left(s_{i}\right)}-\underline{p_{B}\left(s_{i}\right)}\right|^{2}+\frac{1}{2}\left|\overline{p_{A}\left(s_{i}\right)}-\overline{p_{B}\left(s_{i}\right)}\right|^{2}+\frac{1}{4 \pi^{2}}\left|q_{A}\left(s_{i}\right)-q_{B}\left(s_{i}\right)\right|^{2}\right) .}
\end{aligned}
$$

However, these distances are not suitable for localization problem; for example, let $\mu_{A}(s) \equiv 0.01 \cdot e^{j 0.25 \pi}$ and $\mu_{B}(s) \equiv 0.01 \cdot e^{j 1.25 \pi}$; they are very close, as shown in Figure 1 , but we have $D_{H}(A, B)=0.25 n, D_{E}(A, B)=$ $(\sqrt{2 n} / 4), D_{n H}(A, B)=0.25$, and $D_{n E}(A, B)=\sqrt{2} / 4$. This is not consistent with our intuition.
In order to overcome the abovementioned shortcoming, we introduce some new distances for IVCFSs. Let $u=\left[p_{1}, \overline{p_{1}}\right] \cdot e^{j q_{1}}$ and $v=\left[p_{2}, \overline{p_{2}}\right] \cdot e^{j q_{2}}$ be two IVCFVs, and we consider the following distances between $u$ and $v$ :

$$
\begin{aligned}
& d_{H}(u, v)=\max \left(\left|\underline{p_{1}} \cdot e^{j q_{1}}-\underline{p_{2}} \cdot e^{j q_{2}}\right|_{1},\left|\overline{p_{1}} \cdot e^{j q_{1}}-\overline{p_{2}} \cdot e^{j q_{2}}\right|_{1},\left|\overline{p_{1}} \cdot e^{j q_{1}}-\underline{p_{2}} \cdot e^{j q_{2}}\right|_{1},\left|\underline{p_{1}} \cdot e^{j q_{1}}-\overline{p_{2}} \cdot e^{j q_{2}}\right|_{1}\right), \\
& d_{E}(u, v)=\max \left(\left|\underline{p_{1}} \cdot e^{j q_{1}}-\underline{p_{2}} \cdot e^{j q_{2}}\right|_{2},\left|\overline{p_{1}} \cdot e^{j q_{1}}-\overline{p_{2}} \cdot e^{j q_{2}}\right|_{2},\left|\overline{p_{1}} \cdot e^{j q_{1}}-\underline{p_{2}} \cdot e^{j q_{2}}\right|_{2},\left|\underline{p_{1}} \cdot e^{j q_{1}}-\overline{p_{2}} \cdot e^{j q_{2}}\right|_{2}\right),
\end{aligned}
$$

where $|a-b|_{1}$ and $|a-b|_{2}$ represent traditional Hamming and Euclidean distances of complex numbers $a, b \in \mathbb{C}$, respectively.
Based on the above formulas, we define some distances of IVCFSs, for any $P, Q \in \operatorname{IVCF}(S)$, where $S=\left\{s_{1}, s_{2}, \ldots, s_{n}\right\}$, we have the following.

(i) The Hamming distance:

$$
\begin{aligned}
h(P, Q)= & \sum_{s \in S} \max \left(\left|\underline{p_{P}(s)} \cdot e^{j q_{P}(s)}-\underline{p_{Q}(s)} \cdot e^{j q_{Q}(s)}\right|_{1},\left|\underline{p_{P}(s)} \cdot e^{j q_{P}(s)}-\overline{p_{Q}(s)} \cdot e^{j q_{Q}(s)}\right|_{1},\right. \\
& \left.\cdot\left|\overline{p_{P}(s)} \cdot e^{j q_{P}(s)}-\underline{p_{Q}(s)} \cdot e^{j q_{Q}(s)}\right|_{1},\left|\overline{p_{P}(s)} \cdot e^{j q_{P}(s)}-\overline{p_{Q}(s)} \cdot e^{j q_{Q}(s)}\right|_{1}\right) .
\end{aligned}
$$

(ii) The normalized Hamming distance:

$$
\begin{aligned}
l(P, Q)= & \frac{1}{2 n} \sum_{s \in S} \max \left(\left|\underline{p_{P}(s)} \cdot e^{j q_{P}(s)}-\underline{p_{Q}(s)} \cdot e^{j q_{Q}(s)}\right|_{1},\left|\underline{p_{P}(s)} \cdot e^{j q_{Q}(s)}-\overline{p_{Q}(s)} \cdot e^{j q_{Q}(s)}\right|_{1},\right. \\
& \left.\cdot\left|\overline{p_{P}(s)} \cdot e^{j q_{P}(s)}-\underline{p_{Q}(s)} \cdot e^{j q_{Q}(s)}\right|_{1}, \overline{p_{P}(s)} \cdot e^{j q_{P}(s)}-\left.\overline{p_{Q}(s)} \cdot e^{j q_{Q}(s)}\right|_{1}\right) .
\end{aligned}
$$

(iii) The Euclidean distance:

$$
\begin{aligned}
e(P, Q)= & \sum_{s \in S} \max \left(\left|\underline{p_{P}(s)} \cdot e^{j q_{P}(s)}-\underline{p_{Q}(s)} \cdot e^{j q_{Q}(s)}\right|_{2}\left|\underline{p_{P}(s)} \cdot e^{j q_{Q}(s)}-\overline{p_{Q}(s)} \cdot e^{j q_{Q}(s)}\right|_{2},\right. \\
& \left.\cdot\left|\overline{p_{P}(s)} \cdot e^{j q_{P}(s)}-\underline{p_{Q}(s)} \cdot e^{j q_{Q}(s)}\right|_{2}, \overline{p_{P}(s)} \cdot e^{j q_{P}(s)}-\left.\overline{p_{Q}(s)} \cdot e^{j q_{Q}(s)}\right|_{2}\right) .
\end{aligned}
$$


(iv) The normalized Euclidean distance:

$$
\begin{array}{r}
q(P, Q)=\frac{1}{2 n} \sum_{s \in S} \max \left(\left|\underline{p_{P}(s)} \cdot e^{j q_{P}(s)}-\underline{p_{Q}(s)} \cdot e^{j q_{Q}(s)}\right|_{2},\left|\underline{p_{P}(s)} \cdot e^{j q_{Q}(s)}-\overline{p_{Q}(s)} \cdot e^{j q_{Q}(s)}\right|_{2},\right. \\
\left.\left|\overline{p_{P}(s)} \cdot e^{j q_{P}(s)}-\underline{p_{Q}(s)} \cdot e^{j q_{Q}(s)}\right|_{2}, \overline{p_{P}(s)} \cdot e^{j q_{P}(s)}-\left.\overline{p_{Q}(s)} \cdot e^{j q_{Q}(s)}\right|_{2}\right) .
\end{array}
$$

Lemma 1. Let $p_{1}, p_{2}, q_{1}, q_{2}, r_{1}, r_{2} \geq 0$, and if $p_{1}+q_{1} \geq r_{1}$ and $p_{2}+q_{2} \geq r_{2}$, then $\max \left(p_{1}, p_{2}\right)+\max \left(q_{1}, q_{2}\right) \geq \max \left(r_{1}, r_{2}\right)$.

Proof. It is easy from $\max \left(p_{1}, p_{2}\right)+\max \left(q_{1}, q_{2}\right) \geq p_{1}+$ $q_{1} \geq r_{1}$ and $\max \left(p_{1}, p_{2}\right)+\max \left(q_{1}, q_{2}\right) \geq p_{2}+q_{2} \geq r_{2}$.

Theorem 1. The above-defined functions $h(P, Q), l(P, Q)$, $e(P, Q), q(P, Q)$ are distances of IVCFSs.
Proof

(1) It is clear that $h(P, Q) \geq 0$ and $h(Q, Q)=0$ for any $P, Q \in \operatorname{IVCF}(S)$. If $h(P, Q)=0$, by the definition of function $h$, we have $p_{P}(s) \cdot e^{j q_{P}(s)}=p_{Q}(s) \cdot e^{j q_{Q}(s)}$ and $\overline{p_{P}(s)} \cdot e^{j q_{P}(s)}=\overline{p_{Q}(s)} \cdot e^{j q_{Q}(s)}$ for all $s \in S$, then $P=Q$.

(2) For any $P, Q \in \operatorname{IVCF}(S)$,

$$
\begin{aligned}
h(P, Q)= & \sum_{s \in S} \max \left(\left|\underline{p_{P}(s)} \cdot e^{j q_{P}(s)}-\underline{p_{Q}(s)} \cdot e^{j q_{Q}(s)}\right|_{1}, \underline{p_{P}(s)} \cdot e^{j q_{P}(s)}-\left.\overline{p_{Q}(s)} \cdot e^{j q_{Q}(s)}\right|_{1},\right. \\
& \left.\cdot\left|\overline{p_{P}(s)} \cdot e^{j q_{P}(s)}-\underline{p_{Q}(s)} \cdot e^{j q_{Q}(s)}\right|_{1},\left|\overline{p_{P}(s)} \cdot e^{j q_{P}(s)}-\overline{p_{Q}(s)} \cdot e^{j q_{Q}(s)}\right|_{1}\right) \\
= & \sum_{s \in S} \max \left(\left|\underline{p_{Q}(s)} \cdot e^{j q_{Q}(s)}-\underline{p_{P}(s)} \cdot e^{j q_{P}(s)}\right|_{1}\left|\underline{p_{Q}(s)} \cdot e^{j q_{Q}(s)}-\overline{p_{P}(s)} \cdot e^{j q_{P}(s)}\right|_{1},\right. \\
& \left.\cdot\left|\overline{p_{Q}(s)} \cdot e^{j q_{Q}(s)}-\underline{p_{P}(s)} \cdot e^{j q_{P}(s)}\right|_{1},\left|\overline{p_{Q}(s)} \cdot e^{j q_{Q}(s)}-\overline{p_{P}(s)} \cdot e^{j q_{P}(s)}\right|_{1}\right) \\
= & h(Q, P) .
\end{aligned}
$$

(3) Since for any $p, q, r \in \mathbb{C}$, we have $|p-q|_{1}+|q-r|_{1} \geq|p-r|_{1}$. Then, for any $s \in S$,

$$
\begin{aligned}
\left|\underline{p_{P}(s)} \cdot e^{j q_{P}(s)}-\underline{p_{Q}(s)} \cdot e^{j q_{Q}(s)}\right|_{1}+\left|\underline{p_{Q}(s)} \cdot e^{j q_{Q}(s)}-\underline{p_{R}(s)} \cdot e^{j q_{R}(s)}\right|_{1} \\
\geq\left|\underline{p_{P}(s)} \cdot e^{j q_{P}(s)}-\underline{p_{R}(s)} \cdot e^{j q_{R}(s)}\right|_{1}, \\
\quad \cdot\left|\underline{p_{P}(s)} \cdot e^{j q_{P}(s)}-\overline{p_{Q}(s)} \cdot e^{j q_{Q}(s)}\right|_{1}+\left|\underline{p_{Q}(s)} \cdot e^{j q_{Q}(s)}-\overline{p_{R}(s)} \cdot e^{j q_{R}(s)}\right|_{1} \\
\geq\left|\underline{p_{P}(s)} \cdot e^{j q_{P}(s)}-\overline{p_{R}(s)} \cdot e^{j q_{R}(s)}\right|_{1}, \\
\quad \cdot\left|\overline{p_{P}(s)} \cdot e^{j q_{P}(s)}-\underline{p_{Q}(s)} \cdot e^{j q_{Q}(s)}\right|_{1}+\left|\overline{p_{Q}(s)} \cdot e^{j q_{Q}(s)}-\underline{p_{R}(s)} \cdot e^{j q_{R}(s)}\right|_{1} \\
\geq\left|\overline{p_{P}(s)} \cdot e^{j q_{P}(s)}-\underline{p_{R}(s)} \cdot e^{j q_{R}(s)}\right|_{1}, \\
\quad \cdot\left|\overline{p_{P}(s)} \cdot e^{j q_{P}(s)}-\overline{p_{Q}(s)} \cdot e^{j q_{Q}(s)}\right|_{1}+\left|\overline{p_{Q}(s)} \cdot e^{j q_{Q}(s)}-\overline{p_{R}(s)} \cdot e^{j q_{R}(s)}\right|_{1} \\
\geq\left|\overline{p_{P}(s)} \cdot e^{j q_{P}(s)}-\overline{p_{R}(s)} \cdot e^{j q_{R}(s)}\right|_{1} \cdot
\end{aligned}
$$


Using Lemma 1, we get

$$
\begin{aligned}
& \max \left(\left|\underline{p_{P}(s)} \cdot e^{j q_{P}(s)}-\underline{p_{Q}(s)} \cdot e^{j q_{Q}(s)}\right|_{1},\left|\underline{p_{P}(s)} \cdot e^{j q_{P}(s)}-\overline{p_{Q}(s)} \cdot e^{j q_{Q}(s)}\right|_{1},\right. \\
&\left.\quad \cdot\left|\overline{p_{P}(s)} \cdot e^{j q_{P}(s)}-\underline{p_{Q}(s)} \cdot e^{j q_{Q}(s)}\right|_{1},\left|\overline{p_{P}(s)} \cdot e^{j q_{P}(s)}-\overline{p_{Q}(s)} \cdot e^{j q_{Q}(s)}\right|_{1}\right) \\
& \quad+\max \left(\left|\underline{p_{R}(s)} \cdot e^{j q_{R}(s)}-\underline{p_{Q}(s)} \cdot e^{j q_{Q}(s)}\right|_{1}\left|\underline{p_{R}(s)} \cdot e^{j q_{R}(s)}-\overline{p_{Q}(s)} \cdot e^{j q_{Q}(s)}\right|_{1},\right. \\
&\left.\quad \cdot\left|\overline{p_{R}(s)} \cdot e^{j q_{R}(s)}-\underline{p_{Q}(s)} \cdot e^{j q_{Q}(s)}\right|_{1},\left|\overline{p_{R}(s)} \cdot e^{j q_{R}(s)}-\overline{p_{Q}(s)} \cdot e^{j q_{Q}(s)}\right|_{1}\right) \\
& \geq \max \left(\left|\underline{p_{P}(s)} \cdot e^{j q_{P}(s)}-\underline{p_{R}(s)} \cdot e^{j q_{R}(s)}\right|_{1}\left|\underline{p_{P}(s)} \cdot e^{j q_{P}(s)}-\overline{p_{R}(s)} \cdot e^{j q_{R}(s)}\right|_{1},\right. \\
&\left.\cdot\left|\overline{p_{P}(s)} \cdot e^{j q_{P}(s)}-\underline{p_{R}(s)} \cdot e^{j q_{R}(s)}\right|_{1},\left|\overline{p_{P}(s)} \cdot e^{j q_{P}(s)}-\overline{p_{R}(s)} \cdot e^{j q_{R}(s)}\right|_{1}\right) .
\end{aligned}
$$

Then,

$$
\begin{aligned}
& \sum_{s \in S} \max \left(\left|\underline{p_{P}(s)} \cdot e^{j q_{P}(s)}-\underline{p_{Q}(s)} \cdot e^{j q_{Q}(s)}\right|_{1},\left|\underline{p_{P}(s)} \cdot e^{j q_{P}(s)}-\overline{p_{Q}(s)} \cdot e^{j q_{Q}(s)}\right|_{1},\right. \\
&\left.\quad \cdot\left|\overline{p_{P}(s)} \cdot e^{j q_{P}(s)}-\underline{p_{Q}(s)} \cdot e^{j q_{Q}(s)}\right|_{1},\left|\overline{p_{P}(s)} \cdot e^{j q_{P}(s)}-\overline{p_{Q}(s)} \cdot e^{j q_{Q}(s)}\right|_{1}\right) \\
& \quad+\sum_{s \in S} \max \left(\left|\underline{p_{R}(s)} \cdot e^{j q_{R}(s)}-\underline{p_{Q}(s)} \cdot e^{j q_{Q}(s)}\right|_{1},\left|\underline{p_{R}(s)} \cdot e^{j q_{R}(s)}-\overline{p_{Q}(s)} \cdot e^{j q_{Q}(s)}\right|_{1},\right. \\
& \geq\left.\left|\overline{p_{R}(s)} \cdot e^{j q_{R}(s)}-\underline{p_{Q}(s)} \cdot e^{j q_{Q}(s)}\right|_{1},\left|\overline{p_{R}(s)} \cdot e^{j q_{R}(s)}-\overline{p_{Q}(s)} \cdot e^{j q_{Q}(s)}\right|_{1}\right) \\
& \sum_{s \in S} \max \left(\left|\underline{p_{P}(s)} \cdot e^{j q_{P}(s)}-\underline{p_{R}(s)} \cdot e^{j q_{R}(s)}\right|_{1},\left|\underline{p_{P}(s)} \cdot e^{j q_{P}(s)}-\overline{p_{R}(s)} \cdot e^{j q_{R}(s)}\right|_{1},\right. \\
&\left.\cdot\left|\overline{p_{P}(s)} \cdot e^{j q_{P}(s)}-\underline{p_{R}(s)} \cdot e^{j q_{R}(s)}\right|_{1}\left|\overline{p_{P}(s)} \cdot e^{j q_{P}(s)}-\overline{p_{R}(s)} \cdot e^{j q_{R}(s)}\right|_{1}\right) .
\end{aligned}
$$

Thus, we can obtain $h(P, Q)=)+h(Q, R) \geq h(P, R)$. Analogously, we can get that the functions $l(P, Q), e(P, Q), q(P, Q)$ are distances.

Thus, we have defined some new distances between IVCFSs. Compared with equations (2)-(4), the distances of IVCFSs in [22], our distances are conformable to human's intuitive receipt when IVCFSs are used to express locative information, such as " $0.5 \mathrm{~km}-0.6 \mathrm{~km}$, east" and " $0.5 \mathrm{~km}-0.7 \mathrm{~km}$, northwest" about the targets. Clearly, our distances can overcome the drawback of CFS' distances as given in Introduction; i.e., the distance between $\varepsilon \cdot e^{j 0.25 \pi}$ and $\varepsilon \cdot e^{j 1.25 \pi}$ is small when $\varepsilon$ is small.

Theorem 2. Let $S=\left\{s_{1}, s_{2}, \ldots, s_{n}\right\}$, for any $P, Q \in \operatorname{IVCF}(S)$, the following inequalities hold:
(1) $0 \leq h(P, Q) \leq 2 \sqrt{2} n$.
(2) $0 \leq l(P, Q) \leq \sqrt{2}$.
(3) $0 \leq e(P, Q) \leq 2 n$.
(4) $0 \leq q(P, Q) \leq 1$.

Proof. For any complex numbers $a, b \in\{c \in \mathbb{C}|| c \mid \leq 1\}$, we have $0 \leq|a-b|_{1} \leq 2 \sqrt{2}$ and $0 \leq|a-b|_{2} \leq 2$, and hence, $h(P, Q) \leq \sum_{1}^{n} 2 \sqrt{2} \leq 2 \sqrt{2} n, \quad e(P, Q) \leq \sum_{1}^{n} 2 \leq 2 n, \quad l(P, Q) \leq$ $(1 / 2 n) \sum_{1}^{n} 2 \sqrt{2} \leq \sqrt{2}$, and $q(P, Q) \leq(1 / 2 n) \sum_{1}^{n} 2 \leq 1$.

In general, we use $e(A, B)$ to measure the distance between two targets $A$ and $B$. But when targets are in the city, $h(A, B)$ is viewed as the city block distance may be more reasonable. Figure 3 shows an instance of the difference between two distances.

Based on the relations among IVCFS, IVFS, CFS, and FS, we give the comparison of our proposed distances of IVCFSs with IVFS, CFS, and FS. Based on the reduction of IVCFSs, the comparison results are shown in Remarks $1-3$.

Remark 1. When IVCFSs are reduced to CFSs, the abovedefined functions (4)-(7) are distances for CFSs based on traditional Hamming and Euclidean distances of complex numbers defined as follows: 


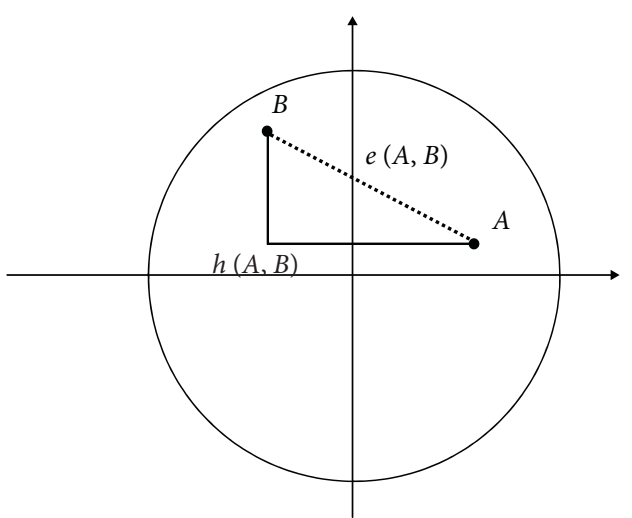

Figure 3: $h(A, B)$ versus $e(A, B)$.

$$
\begin{aligned}
& h(P, Q)=\sum_{s \in S}\left(\left|\psi_{P}(s)-\psi_{Q}(s)\right|_{1}\right), \\
& l(P, Q)=\frac{1}{2 n} \sum_{s \in S}\left(\left|\psi_{P}(s)-\psi_{Q}(s)\right|_{1}\right), \\
& e(P, Q)=\sum_{s \in S}\left(\left|\psi_{P}(s)-\psi_{Q}(s)\right|_{2}\right), \\
& q(P, Q)=\frac{1}{2 n} \sum_{s \in S}\left(\left|\psi_{P}(s)-\psi_{Q}(s)\right|_{2}\right) .
\end{aligned}
$$

Remark 2. When IVCFSs are reduced to IVFSs, the abovedefined functions (4)-(7) are distances for IVFSs based on Hausdorff metric defined as follows:

$$
\begin{aligned}
h(P, Q) & =e(P, Q) \\
& =\sum_{s \in S} \max \left(\left|\underline{p_{P}(s)}-\underline{p_{Q}(s)}\right|,\left|\overline{p_{P}(s)}-\overline{p_{Q}(s)}\right|\right), \\
l(P, Q) & =q(P, Q) \\
& =\frac{1}{2 n} \sum_{s \in S} \max \left(\left|\underline{p_{P}(s)}-\underline{p_{Q}(s)}\right|,\left|\overline{p_{P}(s)}-\overline{p_{Q}(s)}\right|\right) .
\end{aligned}
$$

Remark 3. When IVCFSs are reduced to FSs, the abovedefined functions (4)-(7) are distances for FSs as follows:

$$
\begin{aligned}
& h(P, Q)=e(P, Q)=\sum_{s \in S}\left(\left|p_{P}(s)-p_{Q}(s)\right|\right), \\
& l(P, Q)=q(P, Q)=\frac{1}{2 n} \sum_{s \in S}\left(\left|p_{P}(s)-p_{Q}(s)\right|\right) .
\end{aligned}
$$

Example 1. Let $S=\left\{s_{1}, s_{2}, s_{3}, s_{4}\right\}, P, Q \in \operatorname{IVCF}(S)$ are defined as

Then, by equations (4)-(7), we have

$$
\begin{gathered}
h(P, Q) \approx 3.8253, \\
l(P, Q) \approx 0.4782, \\
e(P, Q) \approx 3.1526, \\
q(P, Q) \approx 0.3941 .
\end{gathered}
$$

\section{Rotational Invariance and Reflectional Invariance}

Let $P \in \operatorname{IVCF}(S)$, then the rotation of $P$ by $\alpha$ radians, denoted $\operatorname{Rot}_{\theta}(P)$, is defined as

$$
\operatorname{Rot}_{\alpha}\left(\mu_{P}(s)\right)=\left[\underline{p_{P}(s)}, \overline{p_{P}(s)}\right] \cdot e^{j\left(q_{P}(s)+\alpha\right)} .
$$

And the reflection of $P$, denoted $\operatorname{Ref}(P)$, is defined as

$$
\operatorname{Ref}\left(\mu_{P}(s)\right)=\left[\underline{p_{P}(s)}, \overline{p_{P}(s)}\right] \cdot e^{j\left(2 \pi-q_{P}(s)\right)} .
$$

Dai et al. [22] gave the following definitions for distance measures between IVCFSs.

Definition 2 (see [22]). Let $d$ is a distance for IVCFSs, and $d$ is rotationally invariant if

$$
d\left(\operatorname{Rot}_{\alpha}(P), \operatorname{Rot}_{\alpha}(Q)\right)=d(P, Q),
$$

for any $\alpha$ and $P, Q \in \operatorname{IVCF}(S)$.

Definition 3 (see [22]). Let $d$ is a distance for IVCFSs, and $d$ is reflectionally invariant if

$$
d(\operatorname{Ref}(P), \operatorname{Ref}(Q))=d(P, Q),
$$

for any $P, Q \in \operatorname{IVCF}(S)$.

Theorem 3. The above-defined distances $e, q$ are reflectionally and rotationally invariant. 
TABle 1: Two properties of distance measures between IVCFSs.

\begin{tabular}{lcc}
\hline & Reflectional invariance & Rotational invariance \\
\hline Distances in [22] & $\sqrt{ }$ & $\times$ \\
$h, l$ & $\sqrt{ }$ & $\times$ \\
$e, q$ & $\sqrt{ }$ & $\sqrt{ }$ \\
\hline
\end{tabular}

TABLE 2: Rating values of the alternatives.

\begin{tabular}{lcccc}
\hline & $E_{1}$ & $E_{2}$ & $E_{3}$ & $E_{4}$ \\
\hline$T_{1}$ & {$[0.8,0.9] \cdot e^{j 1.5 \pi}$} & {$[0.8,0.9] \cdot e^{j 1.6 \pi}$} & {$[0.7,0.8] \cdot e^{j 1.5 \pi}$} & {$[0.7,0.9] \cdot e^{j 1.5 \pi}$} \\
$T_{2}$ & {$[0.7,0.8] \cdot e^{j 1.6 \pi}$} & {$[0.7,0.8] \cdot e^{j 1.8 \pi}$} & {$[0.8,0.9] \cdot e^{j 1.7 \pi}$} & {$[0.7,0.8] \cdot e^{j 1.7 \pi}$} \\
$T_{3}$ & {$[0.1,0.2] \cdot e^{j 0.9 \pi}$} & {$[0.1,0.3] \cdot e^{j 1 \pi}$} & {$[0.1,0.2] \cdot e^{j 1 \pi}$} & {$[0.2,0.3] \cdot e^{j 0.9 \pi}$} \\
$T_{4}$ & {$[0.8,0.9] \cdot e^{j 0.6 \pi}$} & {$[0.7,0.8] \cdot e^{j 0.6 \pi}$} & {$[0.6,0.8] \cdot e^{j 0.4 \pi}$} & {$[0.6,0.8] \cdot e^{j 0.6 \pi}$} \\
\hline
\end{tabular}

Proof. It is easy from the facts that traditional Euclidean distance between complex numbers is reflectionally and rotationally invariant.

Theorem 4. The above-defined distances $h, l$ are reflectionally invariant, but not rotationally invariant.

Proof

(1) It is easy from the fact that $|(a+b j)-(c+d j)|_{1}=$ $|a-c|+|b-d|=|(a-b j)-(c-d j)|_{1}$ for any complex numbers $a+b j$ and $c+d j$. Thus,
TABLe 3: Distance results.

\begin{tabular}{lcccc}
\hline & $h$ & $l$ & $e$ & $q$ \\
\hline$T_{1}$ & 7.1779 & 0.8972 & 5.0911 & 0.6364 \\
$T_{2}$ & 4.7348 & 0.5919 & 3.3717 & 0.4215 \\
$T_{3}$ & 6.13 & 0.7663 & 4.9805 & 0.6226 \\
$T_{4}$ & 6.6132 & 0.8267 & 5.5305 & 0.6913 \\
\hline
\end{tabular}

$\left|r_{1} \cdot e^{j s_{1}}-r_{2} \cdot e^{j s_{2}}\right|_{1}=r_{1} \cdot e^{j-s_{1}}-\left.r_{2} \cdot e^{-j s_{2}}\right|_{1}$. Then, for any $P, Q \in \operatorname{IVCF}(S)$,

$$
\begin{aligned}
h(\operatorname{Ref}(P), \operatorname{Ref}(Q))= & \sum_{s \in S} \max \left(\left|\underline{p_{P}(s)} \cdot e^{-j q_{P}(s)}-\underline{p_{Q}(s)} \cdot e^{j-q_{Q}(s)}\right|_{1},\left|\underline{p_{P}(s)} \cdot e^{-j q_{Q}(s)}-\overline{p_{Q}(s)} \cdot e^{-j q_{Q}(s)}\right|_{1},\right. \\
& \left.\left|\overline{p_{P}(s)} \cdot e^{j-q_{P}(s)}-\underline{p_{Q}(s)} \cdot e^{-j q_{Q}(s)}\right|_{1}\left|\overline{p_{P}(s)} \cdot e^{j-q_{P}(s)}-\overline{p_{Q}(s)} \cdot e^{-j q_{Q}(s)}\right|_{1}\right) \\
= & \sum_{s \in S} \max \left(\left|\underline{p_{P}(s)} \cdot e^{j q_{P}(s)}-\underline{p_{Q}(s)} \cdot e^{j q_{Q}(s)}\right|_{1}\left|\underline{p_{P}(s)} \cdot e^{j q_{Q}(s)}-\overline{p_{Q}(s)} \cdot e^{j q_{Q}(s)}\right|_{1},\right. \\
& \left.\left|\overline{p_{P}(s)} \cdot e^{j q_{P}(s)}-\underline{p_{Q}(s)} \cdot e^{j q_{Q}(s)}\right|_{1},\left|\overline{p_{P}(s)} \cdot e^{j q_{P}(s)}-\overline{p_{Q}(s)} \cdot e^{j q_{Q}(s)}\right|_{1}\right) \\
= & h(P, Q) .
\end{aligned}
$$

Similarly, we can get $l(\operatorname{Ref}(P), \operatorname{Ref}(Q))=l(P, Q)$.

(2) Let $\alpha=\pi / 4$, then we have $|(1+0 j)-(0+1 j)|_{1}=$ $|1-0|+|0-1|=2$, but $\mid(1+0 j) e^{j \pi / 4}-(0+1 j)$ $\left.e^{j \pi / 4}\right|_{1}=|(\sqrt{2} / 2+j \sqrt{2} / 2)-((-\sqrt{2} / 2)+j \sqrt{2} / 2)|_{1}=$ $\sqrt{2}$. Thus, the above-defined distances $h, l$ are not rotationally invariant.

\section{Numerical Example for Decision-Making}

In real life, we may get some answers such as " $0.5 \mathrm{~km}-0.6 \mathrm{~km}$, east" and " $0.5 \mathrm{~km}-0.7 \mathrm{~km}$, northwest" about the targets. These answers can be represented in terms of IVCFSs. Now, we consider a decision-making problem in the environment of IVCFSs. Assume that the ideal target is 1 , and there are four alternatives $\left(T_{1}, T_{2}, T_{3}, T_{4}\right)$. Then, rating values of these alternatives are given by five natives $\left(E_{1}, E_{2}, E_{3}, E_{4}\right)$, and then, we try to find the nearest alternative. The corresponding rating values of alternatives given by natives are shown in Table 1.

Now, we compute the distance between the ideal target and $A_{i}(i=1,2,3,4)$ based on the distance functions (5)-(8). The results are shown in Table 2 .

Here, we use the technique for order preference by similarity to an ideal solution (TOPSIS) [28] for decision-making. Based on the TOPSIS method, we try to find the nearest alternative to the ideal target, and thus, the best alternative is the one with the nearest distance to the ideal target.

The results are shown in Table 3 , in which $T_{i}>T_{k}$ means $T_{i}$ is nearer than $T_{k}$ for the ideal target. Then, as we can see in Table $4, T_{2}$ is the best alternative in this example. 
TABLE 4: Ordering of the alternatives.

\begin{tabular}{lc}
\hline & Ordering \\
\hline$h$ & $T_{2}>T_{3}>T_{4}>T_{1}$ \\
$l$ & $T_{2}>T_{3}>T_{4}>T_{1}$ \\
$e$ & $T_{2}>T_{3}>T_{1}>T_{4}$ \\
$q$ & $T_{2}>T_{3}>T_{1}>T_{4}$ \\
\hline
\end{tabular}

\section{Conclusions}

CFS and IVCFSs are used to describe locative information with uncertainty in some real-world applications; for example, when we ask for directions, we may get answers such as " $0.5 \mathrm{~km}-0.6 \mathrm{~km}$, east", " $0.8 \mathrm{~km}$, West," and " $0.5 \mathrm{~km}-0.7 \mathrm{~km}$, northwest" about the targets. Then, we need to measure the difference between objects and estimate how long it will take to get to the close object. In this case, distances in $[12,16,17,22]$ are not suitable. In this paper, we have presented some new distances for IVCFSs by using traditional Euclidean distance between complex numbers. They are suitable for measuring the distance between objects. We used these distances to deal with the location decision problem under uncertain situations. These distance measures include the Hamming distance $h$, the normalized Hamming distance $l$, the Euclidean distance $e$, and the normalized Euclidean distance $q$. Furthermore, the distances $h$ and $l$ are reflectionally invariant but not rotationally invariant, and distances $e$ and $q$ are both reflectionally and rotationally invariant. Finally, based on these distances, we presented an illustrative example for location decision-making under IVCFS situation.

Note that we give a drawback of distances in $[12,16,17,22]$ from a specific application of IVCFS. Many angles of analysis of distances are needed. In future research, we expect to develop more distances of CFS and its extension from different angles and apply them in different applications, such as engineering, economics, and medicine.

\section{Abbreviations}

FS:

CFS: $\quad$ Complex fuzzy set

IVFS: Interval-valued fuzzy set

IVCFS: Interval-valued complex fuzzy set

IVCFV: Interval-valued complex fuzzy value

IVCF (S): The set of all IVCFSs of $S$

TOPSIS: Technique for order preference by similarity to an ideal solution.

\section{Data Availability}

The data used to support the findings of this study are included in the article.

\section{Conflicts of Interest}

The authors declare that there are no conflicts of interest.

\section{Authors' Contributions}

Haifeng Song, Lvqing Bi, Bo Hu, Yingying Xu, and Songsong Dai contributed equally to this work.

\section{Acknowledgments}

This research was funded by the Zhejiang Provincial Natural Science Foundation of China (Grant nos. LQ21A010001 and LQ21F020001), the Cultivating Science Foundation of Taizhou University (2019PY014), the Agricultural Science and Technology Project of Taizhou (20ny13), and the Opening Foundation of Yulin Research Institute of Big Data (Grant no. 2020YJKY04).

\section{References}

[1] D. Ramot, R. Milo, M. Friedman, and A. Kandel, "Complex fuzzy sets," IEEE Transactions on Fuzzy Systems, vol. 10, no. 2, pp. 171-186, 2002.

[2] Z. Chen, S. Aghakhani, J. Man, and S. Dick, "ANCFIS: a neurofuzzy architecture employing complex fuzzy sets," IEEE Transactions on Fuzzy Systems, vol. 19, no. 2, pp. 305-322, 2011.

[3] C. Li and T.-W. Chiang, "Complex neurofuzzy ARIMA forecasting-A new approach using complex fuzzy sets," IEEE Transactions on Fuzzy Systems, vol. 21, no. 3, pp. 567-584, 2013.

[4] C. Li, T.-W. Chiang, and L.-C. Yeh, "A novel self-organizing complex neuro-fuzzy approach to the problem of time series forecasting," Neurocomputing, vol. 99, pp. 467-476, 2013.

[5] J. Ma, G. Zhang, and J. Lu, "A method for multiple periodic factor prediction problems using complex fuzzy sets," IEEE Transactions on Fuzzy Systems, vol. 20, pp. 32-45, 2012.

[6] D. Rani and H. Garg, "Complex intuitionistic fuzzy power aggregation operators and their applications in multicriteria decision-making," Expert Systems, vol. 35, no. 6, Article ID e12325, 2018.

[7] H. Garg and D. Rani, "Some generalized complex intuitionistic fuzzy aggregation operators and their application to multicriteria decision-making process," Arabian Journal for Science and Engineering, vol. 44, pp. 2679-2698, 2018.

[8] L. Bi, S. Dai, and B. Hu, "Complex fuzzy geometric aggregation operators," Symmetry, vol. 10, no. 7, p. 251, 2018.

[9] L. Bi, S. Dai, B. Hu, and S. Li, "Complex fuzzy arithmetic aggregation operators," Journal of Intelligent \& Fuzzy Systems, vol. 36, no. 3, pp. 2765-2771, 2019.

[10] S. Dai, L. Bi, and B. Hu, "Interval-valued complex fuzzy geometric aggregation operators and their application to decision making," Mathematical Problems in Engineering, vol. 2020, Article ID 9410143, 10 pages, 2020.

[11] D. Ramot, M. Friedman, G. Langholz, and A. Kandel, "Complex fuzzy logic," IEEE Transactions on Fuzzy Systems, vol. 11, no. 4, pp. 450-461, 2003.

[12] G. Zhang, T. S. Dillon, K.-Y. Cai, J. Ma, and J. Lu, "Operation properties and delta-equalities of complex fuzzy sets," International Journal of Approximate Reasoning, vol. 50, pp. 1227-1249, 2009.

[13] B. Hu, L. Bi, and S. Dai, "The orthogonality between complex fuzzy sets and its application to signal detection," Symmetry, vol. 9, no. 9, p. 175, 2017.

[14] B. Hu, L. Bi, and S. Dai, "Approximate orthogonality of complex fuzzy sets and approximately orthogonality 
preserving operators," Journal of Intelligent \& Fuzzy Systems, vol. 37, no. 4, pp. 5025-5030, 2019.

[15] C. Li, T. Wu, and F.-T. Chan, "Self-learning complex neurofuzzy system with complex fuzzy sets and its application to adaptive image noise canceling," Neurocomputing, vol. 94, pp. 121-139, 2012.

[16] B. Hu, L. Bi, S. Dai, and S. Li, "Distances of complex fuzzy sets and continuity of complex fuzzy operations," Journal of Intelligent \& Fuzzy Systems, vol. 35, no. 2, pp. 2247-2255, 2018.

[17] A. U. M. Alkouri and A. R. Salleh, "Linguistic variable, hedges and several distances on complex fuzzy sets," Journal of Intelligent \& Fuzzy Systems, vol. 26, no. 5, pp. 2527-2535, 2014.

[18] S. Dai, "Complex fuzzy ordered weighted distance measures," Iranian Journal of Fuzzy Systems, vol. 17, no. 6, pp. 107-114, 2020.

[19] W. Guo, L. Bi, B. Hu, and S. Dai, "Cosine similarity measure of complex fuzzy sets and robustness of complex fuzzy connectives," Mathematical Problems in Engineering, vol. 2019, Article ID 6716819, 2019.

[20] S. Greenfield, F. Chiclana, and S. Dick, "Interval-valued complex fuzzy logic," in Proceedings of the 2016 IEEE International Conference on Fuzzy Systems (FUZZ-IEEE), Vancouver, BC, Canada, July 2016.

[21] S. Greenfield, F. Chiclana, and S. Dick, "Join and meet operations for interval-valued complex fuzzy logic," in Proceedings of the 2016 Annual Conference of the North American Fuzzy Information Processing Society (NAFIPS), El Paso, TX, USA, October 2016

[22] S. Dai, L. Bi, and B. Hu, "Distance measures between the interval-valued complex fuzzy sets," Mathematics, vol. 7, no. 6, p. 549, 2019.

[23] L. Bi, Z. Zeng, B. Hu, and S. Dai, "Two classes of entropy measures for complex fuzzy sets," Mathematics, vol. 7, p. 96, 2019.

[24] L. A. Zadeh, "The concept of a linguistic variable and its application to approximate reasoning-I," Information Sciences, vol. 8, no. 3, pp. 199-249, 1975.

[25] S. Dick, "Toward complex fuzzy logic," IEEE Transactions on Fuzzy Systems, vol. 13, no. 3, pp. 405-414, 2005.

[26] S. Dai, "A generalization of rotational invariance for complex fuzzy operations," IEEE Transactions on Fuzzy Systems, p. 1, 2020.

[27] S. Dai, "On partial orders in complex fuzzy logic," IEEE Transactions on Fuzzy Systems, vol. 29, no. 3, pp. 698-701, 2021.

[28] C. L. Hwang and K. S. Yoon, Multiple Attribute Decision Making Methods and Applications, Springer, Heidelberg, Germany, 1981. 